Further analyses found no excess risk of cancer in individuals who received blood from donors who developed cancer within 1, 2, 3 or 4 years after donation. In addition, the relative risk of cancer in recipients exposed to precancerous blood was unaffected by the anatomical site of the donor's subsequent cancer.

These data do not support the hypothesis that allogeneic transfusion of cancerous cells can lead to the development of cancer.

Original article Edgren G et al. (2007) Risk of cancer after blood transfusion from donors with subclinical cancer: a retrospective cohort study. Lancet 369: 1724-1730

\section{p-EGFR in skin biopsy samples and EGFR copy number: potential markers of response in HNSCC}

EGFR is commonly overexpressed in head and neck squamous cell carcinomas (HNSCCs) and EGFR inhibition is consequently an important treatment strategy for this cancer. In a recent paper, Agulnik and co-workers have reported the results of a phase I/II trial of the EGFR-inhibitor erlotinib, given in combination with cisplatin, in patients with HNSCC. Using pharmacodynamic tissue analyses, the authors evaluated the utility of a range of potential markers for clinical outcomes.

Fluorescent in situ hybridization of 31 archival tumor samples revealed that the response rate to erlotinib-cisplatin therapy tended to be greater in patients with high EGFR copy number than in those with low EGFR copy number (50\% vs $15 \%$; $P=0.33)$. Only four patients with high EGFR copy numbers were identified, however, limiting the power of the comparison. Response rates were also nonsignificantly higher for tumors with stronger immunohistochemical staining for EGFR, and for those with a greater proportion of cells stained. In nine paired tumor biopsy samples (pretreatment vs 7 days of erlotinib treatment; immunofluorescence analysis), elevated baseline levels of p27 and p-STAT3 significantly predicted longer time to progression and overall survival, as did decreases in p-EGFR (time to progression), p-NFKB (overall survival) and p27 (both). In 32 paired skin biopsy samples, therapy-induced suppression of $p$-EGFR correlated with increased overall survival (10.1 months vs 4.8 months; $P=0.045)$, indicating that analysis of such samples could be used to monitor treatment efficacy in HNSCC.

Original article Agulnik M et al. (2007) Predictive and pharmacodynamic biomarker studies in tumor and skin tissue samples of patients with recurrent or metastatic squamous cell carcinoma of the head and neck treated with erlotinib. J Clin Oncol 25: 2184-2190

\section{Patients presenting with untreated CLL: nomogram predicts survival}

A wide range of measurable parameters have been shown to predict outcomes in chronic lymphocytic leukemia (CLL); however, a notably heterogeneous disease course means that individually these parameters have limited clinical utility. In a recent paper, Wierda et al. present a multivariate model and associated nomogram a graphic representation of the model suitable for use in clinical practice, research and trial design -for prediction of survival in patients who present with untreated CLL.

The investigators analyzed the characteristics of 1,674 patients who presented with CLL at the MD Anderson Cancer Center, Houston, TX, during the period 1981-2004. Univariate analysis identified a wide range of characteristics at presentation that were predictive of overall survival. Subsequent multivariate analysis identified age, sex, absolute lymphocyte count, serum $\beta 2$ microglobulin, number of involved lymph-node groups and Rai stage as independent predictors of survival. A nomogram incorporating these six variables was constructed on the basis of this multivariate model, and was predictive of 5-year and 10-year overall survival, and of median survival time. Age and $\beta 2$ microglobulin level were the two most heavily weighted model variables.

Although the patient population studied was younger than that generally presenting to community practice (median age 58 vs $>65$ years), a high concordance index for patients aged $>65$ years suggests that the nomogram is applicable to a patient population typical of day-to-day practice. Further work will focus on evaluating the role of more-recently identified prognostic factors, such as immunoglobulin heavy-chain status, in this type of model.

Original article Wierda WG et al. (2007) Prognostic nomogram and index for overall survival in previously untreated patients with chronic lymphocytic leukemia. Blood 109: 4679-4685 